\title{
Sums of reciprocals of integers
}

\author{
Simon Davis
}

\author{
8861 Villa La Jolla Drive \#13595
}

La Jolla, CA 92039, United States

Received: 28 August 2018

\begin{abstract}
The sums of reciprocals are demonstrated to diverge for infinite sequences consisting of arbitrarily long arithmetic progressions. It is demonstrated that there may exist sequences that do not include arithmetic progressions of arbitrary length that yield divergent sums.
\end{abstract}

Keywords: Arithmetic sequences, Length, Divergent sums.

2010 Mathematics Subject Classification: 11B05, 11B25, 11P21, 11 Y55.

\section{Introduction}

It is well known that the harmonic sum diverges when the upper limit tends to infinity. Furthermore, existence of an infinite number of primes follows from the divergence of the sum

$$
\sum_{\text {p prime }} \frac{1}{p} \text {. }
$$

Between the set of natural numbers and the primes are arithmetic progressions. Consider the arithmetic sequence $x_{n}=a n+b, a, b>0$. The sum of the reciprocals is

$$
\begin{aligned}
\sum_{n=1}^{\infty} \frac{1}{a n+b} & =\frac{1}{a} \sum_{n=1} \frac{1}{n+\frac{b}{a}} \\
& >\frac{1}{a} \sum_{n=1}^{\left\lfloor\frac{b}{a}\right\rfloor} \frac{1}{2} \frac{1}{\frac{b}{a}}+\frac{1}{a} \sum_{\left\{\frac{b}{a}\right\}}^{\infty} \frac{1}{2 n}=\infty .
\end{aligned}
$$

While the sum of reciprocals of integers in a finite arithmetic sequence would converge, the possibility of considering the necessity of a set of integers with arithmetic progressions of arbitrarily length for divergence of the sum can be considered [4]. 
Reciprocal sums reflect the distribution of number sets. Convergent sums have been bounded for consecutive primes differing by 2 [1], amicable pairs [8] and primitive nondeficient numbers [7] that are consistent with the order estimates for the cardinality of the sets less than a given integer [2].

When these estimates reach $\mathcal{O}\left(\frac{x}{\ln x}\right)$, which is characteristic of the primes, the reciprocal sums may be divergent. Consequently, the connection between the arithmetic sequences in the sumsets and the divergence of the reciprocal sums would clarify the extent of progressions amongst other integer sets including the primes.

Several advances on the characteristics of infinite sets of integers within the natural numbers indicate the computational complexity of this problem. It has been averred that any subset of $\mathbb{N}$ of positive density includes arithmetic sequences of arbitrarily length [3,9]. Given this statement and other results on measures defined over the integer set $\mathbb{Z}_{N}$, the existence of arbitrarily long arithmetic progressions within the primes may be investigated [6]. The results prompt the study of all arithmetic sequences that can occur in an infinite set in the natural numbers and not only those that are required for its construction. Configurations within a integer lattice in the plane could consist of segments representing bounded linear sequences. Nevertheless, the entire set of lines through the integer points, including those traversing different edges, must be examined. The extent of these arithmetic sequences and the existence of transformations of the number sets, so that the progressions have a bounded maximal length, are elaborated in the following sections. It is demonstrated that points in a saw-toothed configuration can be moved such that the number of elements of the linear sequences are decreased. Then the number of steps is estimated, and the feasibility of constructing a set with a finite upper bound for the the lengths of the arithmetic progressions is demonstrated.

\section{Divergence of the sums of reciprocals over sets of integers including arithmetic progressions}

If the recriprocals have denominators that belong to arbitrarily long arithmetic progressions, the sum of reciprocals will contain

$$
\sum_{k_{1} \in S_{1} \subset \mathbb{Z}} \frac{1}{\alpha_{1}+k_{1} \beta_{1}}+\sum_{k_{2} \in S_{2} \subset \mathbb{Z}} \frac{1}{\alpha_{2}+k_{2} \beta_{2}}+\cdots,
$$

where $\left|S_{1} \cup S_{2} \cup \ldots\right|=\infty$. If there is only one set $S_{1}$, the sum diverges trivially. For a finite number of sets, the arithmetic progressions can be ordered so that $\alpha_{N}+k_{N} \beta_{N}$ is largest and infinite. Then $\sum_{k_{N} \in S_{N} \subset \mathbb{Z}} \frac{1}{\alpha_{N}+k_{N} \beta_{N}}=\infty$. There cannot be an infinite number of sums because almost all would contain only a finite number of elements and no arithmetic progression for a one-to-one correspondence with the set of natural numbers. However, the cardinality may be regarded as equal to $\aleph_{0}$. If the sum of reciprocals is defined by a set with no arbitrarily long arithmetic sequence

$$
\sum_{n=1}^{N} \sum_{k_{n} \in S_{N} \subset \mathbb{Z}} \frac{1}{\alpha_{n}+k_{n} \beta_{n}}<\sum_{n=1}^{\infty}\left(\frac{\kappa}{\alpha_{n}}\right) \quad k \text { bounded, } 1<\kappa<B_{\left.\left\{S_{n}\right\}\right\}} .
$$


When the set $\left\{\alpha_{n}\right\}$ forms a random pattern, this sequence only would be bounded by an arithmetic progression. A bounding by an arithmetic progression yields an infinite bound. To derive a finite bound, it is necessary to choose $\alpha_{n}$ to be increasing as $n^{\gamma}, \gamma>1$. There would be no arithmetic progression bounding $\left\{\alpha_{n}\right\}$.

Consider a random sequence increasing linearly. The graph would be that of data points scattered about a linear plot. To fit this scattered data, either a polynomial or an arithmetic progression $k_{n, 0} \alpha_{n, 0}+k_{n, 1} \alpha_{n, 1}+\cdots+k_{n, n-1} \alpha_{n, n-1}$, is used. When the number of data points is infinite, the linear combination has an infinite number of coefficients. The discrete set of points would be given by the values $k_{n, 0}=1, k_{n, i}=0, i \geq 1, k_{n, 0}=0, k_{n, 1}=1, k_{n, i}=0, i \geq 2$. Line segments may be drawn between these points if $k_{n, 0}(t)=(1-t), k_{n, 1}(t)=t, k_{n, i}=0, i \geq 2$, $k_{n, 0}=0, k_{n, 1}=1-t, k_{n, 2}=t, k_{n, i}=0, i \geq 3,0 \leq t \leq 1$. Since the range of $t$ has two integer values, the corresponding arithmetic progressions has two elements. Letting the first coefficient be 1 and replacing $t$ by an integer parameter $n$, it can determined if the data set includes linear sequences with greater lengths.

The two-term arithmetic progression can be described geometrically in the real plane as a line through a lattice of points bounded by two infinite lines of fixed slopes. Let the lattice of integer points between $\beta_{\text {min }} x$ and $\beta_{\text {max }} x$ be L. The existence of an arbitrarily long arithmetic progression is equivalent to the occurrence of an infinite line belonging to $L$ that consists of elements of the sequence of denominators. It is immediate that the sequence of points can be selected so that an infinite number are not located on a single line with a slope between $\beta_{\min }$ and $\beta_{\max }$. If $\mathrm{L}_{n}$ is the subset of points at $x=n$, then $\left|\mathrm{L}_{n}\right|=\left|\beta_{2} n-\beta_{1} n\right|$. An infinite line will cross one point at each $n$, and there are $\left|\beta_{\max } n-\beta_{\min } n\right|-1$ such points. Suppose a line with slope $\beta>\beta_{\max }$ intersects a sequence of integer points representing denominators in the reciprocal sums. Beginning with the point $\left(y_{0}, n_{0}\right)$, when $\beta_{\min } n_{0} \leq y_{0} \leq \beta_{\max } n_{0}$, and the line $y=y_{0}+\beta\left(x-n_{0}\right)$ will intersect the line $y=\beta_{\max } n_{0}$ when

$$
\begin{aligned}
\beta_{\text {max }} x & =y_{0}+\beta\left(x-n_{0}\right) \\
x & =\frac{\beta n_{0}-y_{0}}{\beta-\beta_{\text {min }}}=\frac{y_{0}-\beta n_{0}}{\beta_{\text {min }}-\beta} \geq n_{0},
\end{aligned}
$$

which is finite. The line therefore will have a finite number of points, and the denominators in the reciprocals will comprise a finite set. Similarly, if $\beta<\beta_{\text {min }}$, the line $y=y_{0}+\beta\left(x-n_{0}\right)$ will intersect the line $y=\beta_{\min } n_{0}$ when

$$
\begin{gathered}
\beta_{\text {min }} x=y_{0}+\beta\left(x-n_{0}\right) \\
\frac{y_{0}}{\beta_{\text {min }}-\beta}>x=\frac{\beta n_{0}-y_{0}}{\beta-\beta_{\text {min }}}=\frac{y_{0}-\beta n_{0}}{\beta_{\text {min }}-\beta} \geq n_{0} .
\end{gathered}
$$

Only a finite number of points representing a finite arithmetic progression would be located on this line. Since any infinite line must have a gradient between $\beta_{\min }$ and $\beta_{\max }$, there exist sequences which do not coincide with these lines. The union of such sequences would form saw-toothed trajectories with edges of bounded length.

Suppose that the edges have a maximum length of $L_{\text {max }}$. Then, if the minimum slope of the lines is $\beta_{0}$, the number of elements in each edge does not exceed $\frac{L_{\max }}{\beta_{0}}$. Even though each edge does not represent a sequence with more than $\frac{L_{\max }}{\beta_{0}}$ elements, it could be conjectured that 
arbitrarily long progressions may be constructed from points belonging to a collection of different edges. Each of these progressions might be reduced to finite length by moving the points off the lines formed by these larger sequences.

It is traditional to require that $\beta_{0} \geq 1$ for arithmetic sequences, and, if $\beta_{1}$ is the maximum slope of an edge, the density $\rho$ of the entire set of integers in a saw-toothed trajectory within the natural numbers would satisfy the inequality $1 \geq \frac{1}{\beta_{0}} \geq \rho \geq \frac{1}{\beta_{1}}>0$. By Szemerédi's theorem, the positive density ensures the existence of a arithmetic progressions of arbitrary length amongst the set of integers in the trajectory.

There remains then the mechanism of moving a set of integer points off the saw-toothed trajectory so that the lengths of all of the larger arithmetic sequences become bounded. The divergent reciprocal sums for these sequences would represent counterexamples to the ErdôsTuran conjecture.

\section{The construction of sequences with bounded arithmetic progressions}

The precise form of the result for sets of density $\delta>0$ is the existence of a number $N_{0}(\delta, k)$ such that, if $N \geq N_{0}(\delta, k)$, it is claimed that the set $S \subset \mathbb{Z}_{N}$, with $|S|=\delta N$, contains an arithmetic sequence of length $k$ [9]. A value of $2^{2^{\delta^{-c_{k}}}}$ has been found for $N_{0}(\delta, k)$, where $c_{k}$ can be set equal to $2^{2^{k+9}}$ [5]. For the saw-toothed trajectory with slopes between $\beta_{0}$ and $\beta_{1}$, the upper bound can be selected to be $N_{0}\left(\frac{1}{\beta_{1}}, k\right)=2^{2^{\beta_{1} 2^{2^{k+9}}}}$.

Theorem. There are elements of two-term arithmetic progressions in the saw-toothed trajectory that can be moved so that the sequences in the set have bounded lengths.

Proof. Let $N \geq N_{0}\left(\frac{1}{\beta_{1}}, k\right)$ and $S_{k, i}$ be a progression of length $k$ in $\mathbb{Z}_{N}$. If a point on each of the lines representing sequences of length $k$ is moved away a distance less than $\frac{N_{0}\left(\frac{1}{\beta_{0}}, k\right)-a_{k, i}}{k}$, where $a_{k, i}$ is the initial term of $S_{k, i}$, then the new progression $\tilde{S}_{\ell, i}$ will have a length $\ell<k$. If these points are selected to be near the midpoints of the line segments, the lengths will be equal approximately to $\left\lfloor\frac{k}{2}\right\rfloor$.

Proceeding to the sequences of length $k-1$, the moving of the midpoint would reduce lengths of those progressions to nearly $\frac{k-1}{2}$. After $1+1+1+\cdots+(1+1)+\cdots$ iterations, the sequences have lengths less than or equal to $\frac{k}{4}$, where $n_{r}, 2 \leq r \leq k$, is the number of progressions of length $r$. Fixing some bound $\bar{k}=\frac{k}{2^{b}}$, all progressions will have lengths less than or equal to $\bar{k}$ after $1+1+1+\cdots+(1+1)+\cdots+(1+1+1+1)+\cdots$ steps. The number of steps will be less than $\left(2^{b-1}+2 \cdot 2^{b-2}+\cdots+2^{b-1}\right)=b 2^{b-1}=\frac{k}{2 k} \log _{2} \frac{k}{k}$. More generally, when $\frac{k}{k}$ is non-integer, the upper bound is given by $\left\{\frac{k}{2 k}\right\} \log _{2}\left\{\frac{k}{k}\right\}$. Therefore, the number of steps to reduce the lengths to the limiting value $\bar{k}$ is much less than $|S| \leq \frac{1}{\beta_{1}} N$. Since the numbers $n_{r}$ are constrained by $\sum_{r=2}^{k} n_{r} r \leq \frac{1}{\beta_{1}} N$, it follows that there is sufficient space, $\left(1-\frac{1}{\beta_{1}}\right)\left(\frac{N_{0}\left(\frac{1}{\beta_{0}}, k\right)-a_{\max }}{r}\right)$, amongst 
the integers in $\mathbb{Z}_{N}$ for the selected points to be moved such that the initial sequences are divided into progressions of length less than or equal to $\bar{k}$.

The arithmetic progressions in the new set of integers $\tilde{S}$ may be listed. Clearly, this set will consist of sequences of length less than or equal to $\bar{k}$ amongst the nearly $|S|-\left\{\frac{k}{2 \bar{k}}\right\} \log _{2}\left\{\frac{k}{k}\right\}$ remaining elements that have not been moved. By definition, there would be no progressions of greater length amongst these elements. The $\left\{\frac{k}{2 k}\right\} \log _{2}\left\{\frac{k}{k}\right\}$ points that have been translated within the lattice are not correlated with these points. It is only necessary to determine the progressions amongst the integers which have been moved.

Suppose that the earlier bound with a new length for the progressions $k^{\prime}, N_{0}\left(\delta, k^{\prime}\right)$, is set equal to $\left\{\frac{k}{2 k}\right\} \log _{2}\left\{\frac{k}{k}\right\}$. With $\delta=\frac{1}{\beta_{0}^{\prime}}$ and $\beta_{0}^{\prime}$ being the new lower bound for the slopes of the lines representing the arithmetic sequences,

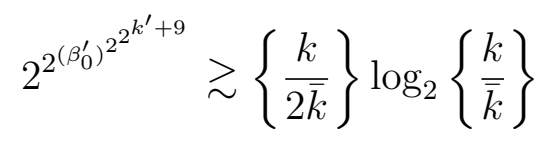

or

$$
k^{\prime}=\left\{\log _{2} \log _{2} \log _{\beta_{0}^{\prime}} \log _{2} \log _{2}\left(\left\{\frac{k}{2 \bar{k}}\right\} \log _{2}\left\{\begin{array}{l}
\frac{k}{\bar{k}} \\
\bar{k}
\end{array}\right)\right\}-9 .\right.
$$

It is not necessary for there exist arithmetic progressions with lengths larger than $k^{\prime}$ in the configuration. If there are sequences with a greater number of elements, additional transformations amongst the $\left\{\frac{k}{2 k}\right\} \log _{2}\left\{\frac{k}{k}\right\}$ points will be sufficient to reduce the lengths to $k^{\prime}$. Following the previous discussion, $\left\{\frac{k^{\prime}}{2 \bar{k}}\right\} \log _{2}\left\{\frac{k^{\prime}}{\bar{k}}\right\}$ would be the maximum number of steps for an initial reduction of the progressions to lengths less than or equal to $\bar{k}$.

The sum of the maximum number of movements of integers in the set $S$ for the arithmetic progressions to have a length less than or equal to $\bar{k}$ is

$$
\left\{\frac{k}{2 \bar{k}}\right\} \log _{2}\left\{\frac{k}{\bar{k}}\right\}+\left\{\frac{k^{\prime}}{2 \bar{k}}\right\} \log _{2}\left\{\frac{k^{\prime}}{\bar{k}}\right\}+\left\{\frac{k^{\prime \prime}}{2 \bar{k}}\right\} \log _{2}\left\{\frac{k^{\prime \prime}}{\bar{k}}\right\}+\left\{\frac{k^{\prime \prime \prime}}{2 \bar{k}}\right\} \log _{2}\left\{\frac{k^{\prime \prime \prime}}{\bar{k}}\right\}+\cdots,
$$

where

$$
\begin{aligned}
& k^{\prime \prime}=\left\{\log _{2} \log _{2} \log _{\beta_{0}^{\prime}} \log _{2} \log _{2}\left(\left\{\frac{k^{\prime}}{2 \bar{k}}\right\} \log _{2}\left\{\frac{k^{\prime}}{\bar{k}}\right\}\right)\right\}-9 \\
& k^{\prime \prime \prime}=\left\{\log _{2} \log _{2} \log _{\beta_{0}^{\prime \prime}} \log _{2} \log _{2}\left(\left\{\frac{k^{\prime \prime}}{2 \bar{k}}\right\} \log _{2}\left\{\frac{k^{\prime \prime}}{\bar{k}}\right\}\right)\right\}-9
\end{aligned}
$$

The series terminates when the value is less than zero. Since $\log _{2} \log _{2} \log _{\beta_{0}^{(s)}} \log _{2} \log _{2} x$ is less than $\log _{16} x$ for $x \geq 1$, there will be a maximum of $s_{\max }$ terms where $s_{\max }$ is the largest integer such that $\log _{16}^{\left(s_{\max }\right)}\left\{\frac{k}{2 \bar{k}}\right\} \ln _{2}\left\{\frac{k}{k}\right\}>9$, and it follows that

$$
\log _{16^{\operatorname{smax}}}\left\{\frac{k}{2 \bar{k}}\right\} \ln _{2}\left\{\begin{array}{l}
\frac{k}{\bar{k}} \\
\}
\end{array}>9\right.
$$




$$
s_{\max }<\log _{16}\left(\left(\left\{\frac{k}{2 \bar{k}}\right\} \log _{2}\left\{\frac{k}{\bar{k}}\right\}\right)^{\frac{1}{9}}\right) .
$$

The sum (3.3) will be less than

$$
\left\{\frac{k}{2 \bar{k}}\right\} \log _{2}\left\{\begin{array}{l}
\frac{k}{\bar{k}} \\
\}
\end{array} s_{\max }<\left\{\frac{k}{2 \bar{k}}\right\} \log _{2}\left\{\begin{array}{l}
\frac{k}{\bar{k}} \\
\}
\end{array} \log _{16}\left(\left\{\frac{k}{2 \bar{k}}\right\} \log _{2}\left\{\begin{array}{l}
\frac{k}{\bar{k}}
\end{array}\right\}\right)^{\frac{1}{9}} .\right.\right.
$$

Again, this upper bound is much less than $|S| \leq \frac{1}{\beta_{1}} N$, and there is enough space to make the remaining moves to construct a configuration with arithmetic progress of maximum length $\bar{k}$.

\section{Conclusion}

It has been demonstrated how integer points on lines intersecting a saw-toothed trajectory with bounded edges can be moved so that arithmetic progressions have a bounded maximum length. The number of steps required for the algorithm is given, and it is found that the entire sum is still considerably less than the lower bound for the number $N$ necessary for existence of arithmetic progressions of length $k$ in a set of positive density in the natural numbers.

It is necessary to verify that the subset of integers in a set $S \subset \mathbb{Z}_{N}$ belonging to arithmetic sequences with $\tilde{k}$ elements, where $\tilde{k}>k$ and $N \geq N_{0}(\delta, k)$, can be moved so that the progressions again would be reduced to a maximum of $k$ elements. This property may only be valid for integers near $N_{0}(\delta, k)$ since $k$ might be increased for larger $N$. Nevertheless, the proof continues to hold since the integers $N_{0}(\delta, k)$ form a sequence that tends to infinity. The estimated bound for $N_{0}(\delta, k)$, for which a precise value is required. incorporates this postulate.

The sets considered in this work have positive density, and the conclusions are consistent with bounds for lengths of arithmetic progressions in finite sets of integers rather than the infinite limit. Consequently, it may follow that a finite version of the result is necessary for the study of arithmetic sequences.

\section{References}

[1] Crandall, R., \& Pomerance, C. (2005). Prime Numbers: A Computational Perspective, Springer, New York.

[2] Erdős, P. (1934). On the Density of the Abundant Numbers, J. London Math. Soc. Ser. 1, 9, $278-282$.

[3] Erdős, P. (1955). On Amicable Numbers, Publ. Math. Debrecen, 4, 108-111.

[4] Erdős, P., \& Turán, P. (1941). On a Problem of Sidon in Additive Number Theory, and on some Related Problems, J. Lond. Math. Soc., 16, 212-216.

[5] Gowers, W. T. (2001). A new proof of Szemerédi's Theorem, Geometric and Functional Analysis, 11, 465-588. 
[6] Green, B., \& Tao, T. (2008). The Primes contain Arbitrarily Long Arithmetic Progressions, Ann. Math., 167, 481-547.

[7] Lichtman, J. D. (2018). The Reciprocal Sum of Primitive Deficient Numbers, arXiv.1801.01925.

[8] Pomerance, C. (1981). On the Distribution of the Amicable Numbers, II. J. Reine Angew. Math., 325, 183-188.

[9] Szemerédi, E. (1975). On Sets of Integers containing no $k$ Elements in Arithmetic Progressions, Arith. Acta, 27, 299-345. 